\title{
Spin-orbit effects in a triple quantum dot shuttle
}

\author{
J. Villavicencio, ${ }^{1, *}$ I. Maldonado, ${ }^{2}$ E. Cota,${ }^{3, \dagger}$ and G. Platero ${ }^{4, \dagger}$ \\ ${ }^{1}$ Facultad de Ciencias, Universidad Autónoma de Baja California, Apartado Postal 1880, 22800 Ensenada, Baja California, México \\ ${ }^{2}$ Avenida Del Fresno 188, Código Postal 22819, Ensenada, B.C., México \\ ${ }^{3}$ Centro de Nanociencias y Nanotecnología, Universidad Nacional Autónoma de México, \\ Apartado Postal 356, 22860 Ensenada, B.C., México \\ ${ }^{4}$ Instituto de Ciencia de Materiales de Madrid (CSIC), Cantoblanco, 28049 Madrid, Spain
}

(Received 14 April 2013; revised manuscript received 12 September 2013; published 10 December 2013)

\begin{abstract}
Spin transport properties of a triple quantum dot shuttle system are analyzed in the presence of spin-orbit interaction. We calculate the spin current through the device as a function of the bias voltage detuning. We find that new resonances in the current through the shuttle appear due to the presence of spin-orbit interaction, and the states involved in the conduction process are identified. Also, spin current polarization is analyzed as a function of tunnel coupling and detuning, and conditions are found where the device can act as a spin filter.
\end{abstract}

DOI: 10.1103/PhysRevB.88.245305

PACS number(s): 73.63.Kv, 03.65.Vf, 03.65.Xp, 73.21.La

\section{INTRODUCTION}

Transport properties of electrons in triple quantum dot (TQD) systems have become the subject of intense investigation recently, due mainly to their potential applications in quantum information. TQDs represent the next level of complexity in the study of the properties of coupled quantum bits (qubits). In these systems, charge stability diagrams have been obtained, ${ }^{1}$ and entanglement ${ }^{2}$ and interference effects in a triangular setup ${ }^{3}$ have been studied. Coherent manipulation of interacting three-spin states has been demonstrated ${ }^{4}$ and a resonant exchange qubit has been realized in a TQD system. ${ }^{5}$ Recently, it has also been shown that, in a linear TQD system, spin blockade becomes bipolar ${ }^{6,7}$ and that a new quantum coherent mechanism, where the charge is transferred via coherent states from one end of the TQD circuit to the other without involving the center site, becomes relevant. ${ }^{6}$ Charge and spin transport properties in triple dots under magnetic fields have been addressed as well. ${ }^{8-10}$ Oscillating TQDs in linear array configurations have been proposed as shuttle systems. ${ }^{11,14-16}$ AC-driven transport through shuttle devices is also a very interesting topic and has recently been investigated. ${ }^{11}$ There, the interplay between photon and phonon side bands as potential tunneling channels gives rise to new features in the current compared with the static or the undriven case. Experimental realizations of a shuttle system, using a metallic island mounted on a cantilever ${ }^{12}$ or oscillating nanopillars, ${ }^{13}$ have been reported. Here, we focus on spin transport properties through a triple quantum dot shuttle (TQDS), taking into account spin-orbit interaction (SOI). We assume that the Zeeman splitting energy is different in each quantum dot (QD); this can be achieved using QDs with the same $g$ factor and local nanomagnets ${ }^{17}$ or by using QDs with different $g$ factors under a homogeneous magnetic field. ${ }^{18}$ Also, fluctuating Overhauser fields due to hyperfine interaction give rise to Zeeman inhomogeneities. ${ }^{6}$ In the present work, we find new resonances in the spin current through a TQDS due to the presence of SOI. We also discuss the conditions required to obtain spin-polarized currents and spin filtering.

The paper is organized as follows: in Sec. II we present the Hubbard-like Hamiltonian that describes the TQDS system and show how SOI is included in our model. In this section we also present the main features of the reduced density matrix (RDM) formulation used to calculate the electronic current through the system. In Sec. III we analyze the new current features due to SOI and explore the current spin polarization and the spin filter mechanism. Finally, in Sec. IV we present the conclusions.

\section{FORMALISM}

The TQDS system consists of a linear array of three QDs [Fig. 1(a)], where the central dot oscillates between the two outer dots, which are located at fixed positions $\pm x_{0}$. Conditions are such that only one electron is in the system at any given time (Coulomb blockade regime). In the presence of an external magnetic field $B$, the level spin degeneracy is broken, resulting in two energy states at each dot separated by the Zeeman energy $\Delta=|g| \mu_{B} B$, where $g$ is the Landé $g$ factor $(g=-0.44$ for GaAs, for example) and $\mu_{B}$ is the Bohr magneton. The system is modeled by means of a Hubbard-like Hamiltonian,

$$
\hat{H}=\hat{H}_{0}+\hat{H}_{\text {osc }}+\hat{H}_{\text {tun }}+\hat{H}_{\text {leads }}+\hat{H}_{\text {dot-leads }} \text {, }
$$

where the first three terms on the left-hand side of the above equation represent the Hamiltonian of the TQD system including the spin degree of freedom and are defined as

$$
\begin{aligned}
\hat{H}_{0} & =\sum_{m, \sigma} \varepsilon_{m, \sigma}|m, \sigma\rangle\langle m, \sigma|, \quad \hat{H}_{\mathrm{osc}}=\hbar \omega d^{\dagger} d, \\
\hat{H}_{\text {tun }} & =\sum_{m \neq c, \sigma} T_{m, \sigma}(\hat{x})[|m, \sigma\rangle\langle c, \sigma|+\text { H.c. }] .
\end{aligned}
$$

In the above equation, the Hamiltonian $\hat{H}_{0}$ represents the TQD system including the spin degree of freedom. The index $m=L, C, R$ labels each $\mathrm{QD}$, and $\sigma=\uparrow, \downarrow$ corresponds to the spin states. Also, allowing for the possibility of different local magnetic fields at each QD, we have defined the dot energies

$$
\varepsilon_{m, \sigma}=\varepsilon_{m}+\Delta_{m, \sigma},
$$

with $\Delta_{m, \downarrow}=\Delta_{m} / 2$ and $\Delta_{m, \uparrow}=-\Delta_{m} / 2, \Delta_{m}$ being the Zeeman splitting at dot $m$. The energy of the central dot is position dependent and is given by

$$
\varepsilon_{C}(\hat{x})=\left[\varepsilon_{L}-\left(\varepsilon / 2 x_{0}\right)\left(\hat{x}+x_{0}\right)\right],
$$



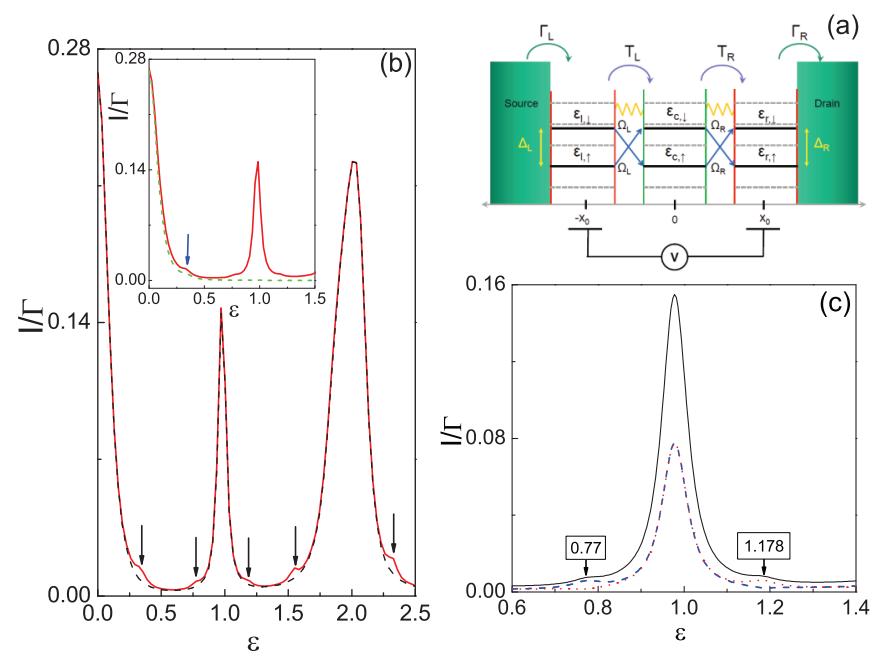

FIG. 1. (Color online) (a) TQDS system with SOI interaction, where the central dot oscillates between two static QDs at fixed position $\pm x_{0}$. There are two energy levels $\varepsilon_{m, \uparrow \downarrow}$ at QD $m$, due to Zeeman splitting $\Delta_{m}$. The interdot tunneling rates, $T_{R}$ and $T_{L}$, as well as those including SOI, $\Omega_{L, R}$, are position dependent. (b) Spin current as a function of detuning $\varepsilon$ for a TQDS system with $\Delta_{m}=0.2$ ( $m=L, C, R$ ) and SOI [solid (red) line], compared with the case without SOI [dashed (black) line]. Note that small satellite peaks emerge as a consequence of the SOI (see arrows). Inset: Comparison of the spin current through the TQDS [solid (red) line] and the static TQD [dashed (green) line] with SOI (see text). (c) Amplification of the total current (solid line) with SOI around $\varepsilon=1$, showing that the shoulder at $\varepsilon \simeq 0.77$ is mainly spin-up current [dashed (blue) line], while the one at $\varepsilon \simeq 1.178$ is predominantly spin-down [dotted (red) line]. Parameter values are $V=0.5, \gamma=0.01, \Gamma=0.05, x_{0}=5.0$, $\alpha=0.4$, and $N=4$.

where it is assumed that it undergoes a Stark shift proportional to its position due to the voltage bias across the device: $\varepsilon=\left(\varepsilon_{L}-\varepsilon_{R}\right)$. In Eq. (4) the position operator $\hat{x}$ is given by $\hat{x}=\Delta x_{\mathrm{zp}}\left(\hat{d}^{\dagger}+\hat{d}\right)$, where $\Delta x_{\mathrm{zp}}=(\hbar / 2 m \omega)^{1 / 2}$ is the zeropoint uncertainty position of the oscillator, and $d^{\dagger}$ and $d$ are creation and destruction boson operators respectively, which obey the following rules when applied to an arbitrary oscillator state $|i\rangle: d^{\dagger}|i\rangle=\sqrt{i+1}|i+1\rangle$ and $d|i\rangle=\sqrt{i}|i-1\rangle$. Also in Eq. (2), the Hamiltonian $\hat{H}_{\text {osc }}$ describes the oscillation of the central QD, with frequency $\omega$, and the Hamiltonian $\hat{H}_{\text {tun }}$ accounts for the interdot tunneling, with position-dependent tunneling rates,

$$
T_{L}(\hat{x})=-V e^{-\alpha\left(x_{0}+\hat{x}\right)}, \quad T_{R}(\hat{x})=-V e^{-\alpha\left(x_{0}-\hat{x}\right)},
$$

where $V$ is the tunneling amplitude and $\alpha$ is the inverse of the tunneling length. The position operator $\hat{x}$ measures the displacement of the vibrational mode. The matrix elements of the tunneling couplings $T_{L(R)}^{i j}=\left\langle i\left|T(\hat{x})_{L(R)}\right| j\right\rangle$ in the oscillator basis states $i$ and $j(i, j=0,1,2, \ldots, N)$ can be calculated analytically following the procedure in Ref. 19, which yields

$$
\begin{aligned}
T_{L}^{i j}= & -V\left[2^{n-m} i ! j !\right]^{1 / 2} e^{-\alpha x_{0}} e^{\zeta^{2} / 4}[-\zeta]^{i-j} \\
& \sum_{k=0}^{j} \frac{\zeta^{2 k}}{2^{k} k !(k+i-j) !(j-k) !}, \quad i \geqslant j,
\end{aligned}
$$

and $T_{R}^{i j}=(-1)^{i-j} T_{L}^{i j}$. In the above equation we have defined $\zeta=\sqrt{2} \Delta x_{z p} \alpha$; the matrix elements for the case $i<j$ are calculated by using the hermiticity of the tunneling couplings.

The remaining two Hamiltonians on the right-hand side of Eq. (1) correspond to the electrons in the leads and are described by

$$
\hat{H}_{\text {leads }}=\sum_{l \in(L, R), k, \sigma} \epsilon_{l, k, \sigma} c_{l, k, \sigma}^{\dagger} c_{l, k, \sigma},
$$

where $c_{l, k, \sigma}^{\dagger}$ and $c_{l, k, \sigma}$ are creation and destruction operators for electrons in the leads, with energy $\epsilon_{l, k, \sigma}$ and spin $\sigma$. The coupling between the leads and the outer dots $(L$ and $R)$ is given by

$$
\hat{H}_{\text {dot-leads }}=\sum_{l \in(L, R), k, \sigma} V_{l, k, \sigma}\left(b_{l, \sigma}^{\dagger} c_{l, k, \sigma}+\text { H.c. }\right),
$$

where $b_{l, \sigma}^{\dagger}$ and $b_{l, \sigma}$ are creation and destruction operators for electrons in the $L / R$ dots, with spin $\sigma$, and $V_{l, k, \sigma}$ are the tunneling matrix elements representing the coupling between the leads and the $L / R$ QDs. In the limit of infinite source-drain voltage, the tunneling rates to the leads $\operatorname{read}^{20}$

$$
\Gamma_{l, \sigma}=\frac{2 \pi}{\hbar} \sum_{k}\left|V_{l, k, \sigma}\right|^{2} \delta\left(\epsilon_{l, \sigma}-\epsilon_{l, k, \sigma}\right),
$$

where $l \in L, R$. Here, for simplicity, we assume spin- and energy-independent transition rates $\Gamma_{L}$ and $\Gamma_{R}$.

To incorporate SOI, we add a new tunneling Hamiltonian which takes into account spin-nonconserving processes, ${ }^{21-24}$

$$
\hat{H}_{\text {tun }}^{\text {SOI }}=\sum_{\sigma \neq \sigma^{\prime}}\left\{i \Omega_{m}(\hat{x})\left[|C, \sigma\rangle\left\langle L, \sigma^{\prime}|+| R, \sigma\right\rangle\left\langle C, \sigma^{\prime}\right|\right]+\text { H.c. }\right\},
$$

where, as before, $\sigma, \sigma^{\prime}=\uparrow, \downarrow$, and interdot tunneling is described by the real parameter $\Omega_{m}(\hat{x})$. This Hamiltonian satisfies hermiticity and time reversal invariance. Following Ref. 22 for the static case, we include SOI phenomenologically and consider that $\Omega_{m}(\hat{x})=T_{m}(\hat{x}) / 10$, which is a good estimation from experiments. ${ }^{25}$

The equation of motion of the system using the standard master equation approach for the $\mathrm{RDM},{ }^{26}$ generalized to include the environment of the oscillator, is given by $\dot{\rho}_{q s}^{i j}=\left[\dot{\rho}_{q s}^{i j}\right]_{\mathrm{RDM}}+\left[\dot{\rho}_{q s}^{i j}\right]_{\mathrm{diss}}$, where $\rho_{q s}^{i j}$ represents the matrix element in the composite basis states $q$ and $s\left[q, s=\left|m^{\prime}, \sigma\right\rangle\right.$, $m^{\prime}=0, L, C, R\left(m^{\prime}=0\right.$ represents the vacuum state), and $\sigma=\uparrow, \downarrow]$ projected on the oscillator basis states $i$ and $j(i, j=$ $0,1,2, \ldots, N)$. The first term in $\dot{\rho}_{q s}^{i j}$ incorporates transitions between the leads and the outer dots ${ }^{27}$ and is given by

$$
\begin{aligned}
& {\left[\dot{\rho}_{q s}^{i j}\right]_{\mathrm{RDM}}} \\
& =-\frac{i}{\hbar}[\hat{H}, \rho]_{q s}^{i j} \\
& \quad+ \begin{cases}\sum_{d \neq q}\left(\Gamma_{d \rightarrow q} \rho_{d d}^{i j}-\Gamma_{q \rightarrow d} \rho_{q q}^{i j}\right), & q=s, \\
-\frac{1}{2}\left(\sum_{d \neq q} \Gamma_{q \rightarrow d}+\sum_{d \neq s} \Gamma_{s \rightarrow d}\right) \rho_{q s}^{i j}, & q \neq s,\end{cases}
\end{aligned}
$$

where the second and third lines represent the time evolution of the occupation probabilities and decoherences, respectively, for the open system. Here, $\Gamma_{k \rightarrow k^{\prime}}$ is the transition rate, as 
defined above, from state $k$ to state $k^{\prime}$ in the system, due to coupling to the leads. The dissipative term in the equation for the density matrix due to coupling to the environment is given by ${ }^{28}$

$$
\begin{aligned}
\dot{\rho}_{\text {diss }}= & -\frac{\gamma}{2} \bar{n}\left[d d^{\dagger} \rho-2 d^{\dagger} \rho d+\rho d d^{\dagger}\right] \\
& -\frac{\gamma}{2}(\bar{n}+1)\left[d^{\dagger} d \rho-2 d \rho d^{\dagger}+\rho d^{\dagger} d\right],
\end{aligned}
$$

where $\gamma$ is the classical damping rate of the oscillator and $\bar{n}$ is the thermal occupation number of the oscillator at temperature $T$. In the low-temperature limit $(\bar{n} \rightarrow 0), \dot{\rho}_{\text {diss }}$ in the composite basis reads

$$
\left[\dot{\rho}_{q s}^{i j}\right]_{\mathrm{diss}}=-\gamma \frac{(i+j)}{2} \rho_{q s}^{i j}+\gamma \sqrt{(i+1)(j+1)} \rho_{q s}^{i+1, j+1} .
$$

By solving these equations we obtain the steady-state electronic current of the system,

$$
I=I^{\uparrow}+I^{\downarrow}=e \Gamma_{R} \sum_{i}\left(\rho_{R \uparrow, R \uparrow}^{i i}+\rho_{R \downarrow, R \downarrow}^{i i}\right),
$$

where $\rho_{R \uparrow, R \uparrow}^{i i}$ and $\rho_{R \downarrow, R \downarrow}^{i i}$ are the occupation probabilities of the right-hand dot for spin-up and spin-down for the $i$ th oscillator state when the steady state has been reached. We define $\varepsilon_{L}=-\varepsilon_{R}=\varepsilon / 2$ and $\Gamma_{R}=\Gamma_{L}=\Gamma$.

\section{RESULTS}

In what follows, we calculate the current through the TQDS for symmetric and asymmetric Zeeman splitting in each QD, taking into account SOI. To explain the current features, we calculate the energy spectrum for the corresponding closed system and identify the states involved in the conduction process for each current peak. Finally, we analyze the current polarization as a function of the detuning $\varepsilon$ and tunnel coupling $\alpha$ and identify a region in this parameter space where the TQDS acts as a spin filter. All the calculations are in units of $\hbar=2 m=1$, and $e=\omega=1$. The energies are given in units of $\omega$, and displacements in units of the zero-point displacement of the oscillator, $\Delta x_{\mathrm{zp}}$.

\section{A. Symmetric case}

First, we consider a TQDS with SOI, for symmetric Zeeman splitting given by $\Delta_{m}=0.2(m=L, C, R)$, and $V=0.5$, $\alpha=0.4, x_{0}=5.0, \gamma=0.01$, and $\Gamma=0.05$. In order to explore the effects of SOI, we show in Figs. 1(b) and 1(c), the current as a function of the detuning $\varepsilon$. We see that without SOI, the current shows resonance peaks at values of $\varepsilon$ which are integer multiples of $\omega$; i.e., additional tunneling channels are opened via phonon absorption or emission. ${ }^{14}$ In the presence of SOI, we obtain additional small satellite peaks [indicated by arrows in Fig. 1(b)] on the sides of the main phonon peaks, with a separation that depends on the Zeeman splitting $\Delta$, which are due to spin-nonconserving tunneling processes. ${ }^{22}$ In order to compare our results with the case of a static TQD with SOI, we have chosen a constant tunneling coupling between the dots by setting $\hat{x}=0$, which yields $T_{L}=T_{R}=-V e^{-\alpha x_{0}} \equiv T_{D}$; thus, for the chosen parameters, we obtain $T_{D}=-0.068$. The comparison is shown in the inset in Fig. 1(b), where we can see that all the features of the current through the symmetric TQDS and static TQD, including the SOI satellite peak, are
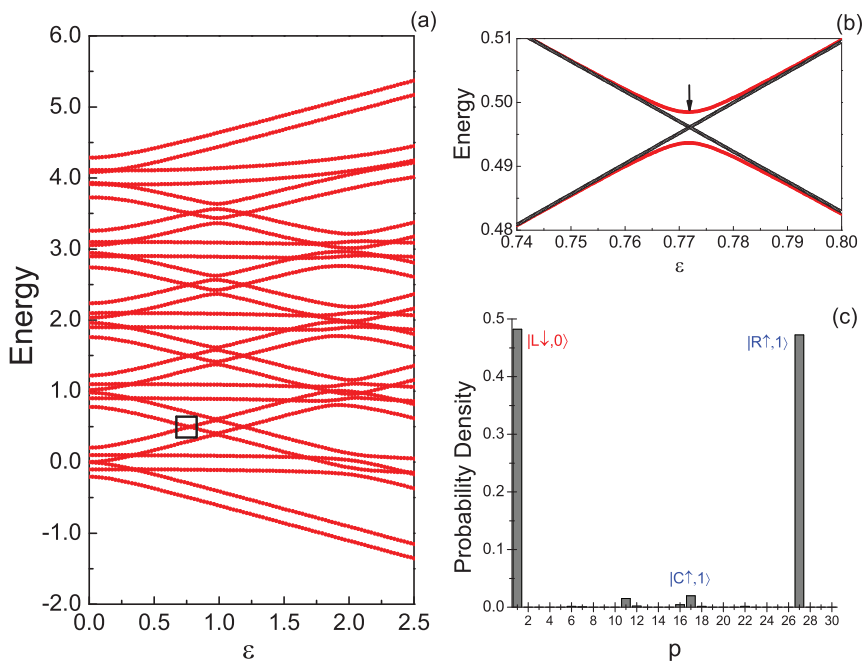

(c)

FIG. 2. (Color online) (a) Eigenvalues for the closed TQDS with symmetric Zeeman splitting including SOI [Fig. 1(b)], as a function of the detuning $\varepsilon$. (b) Amplification around the interval $\varepsilon \in[0.74,0.8]$ [open (black) square in (a)] shows the level anticrossing due to SOI. The case without SOI [open (black) circles] presents an exact crossing instead and is included for comparison. (c) At the level anticrossing region of the spectrum in case (b) we chose a particular eigenvalue at $\varepsilon=0.77$ in order to calculate the corresponding eigenvector and its probability amplitude. The eigenvalue $E=0.498$ is indicated by the black arrow in (b). A satellite peak is observed around the chosen value of detuning $\varepsilon$ [see Fig. 1(c)]. Here $p$ stands for the ordering index of the basis elements, as follows: $\{|L \downarrow, n\rangle\} \leftrightarrow\{1, \ldots, 5\}$, $\{|L \uparrow, n\rangle\} \leftrightarrow\{6, \ldots, 10\},\{|C \downarrow, n\rangle\} \leftrightarrow\{11, \ldots, 15\},\{|C \uparrow, n\rangle\} \leftrightarrow$ $\{16, \ldots, 20\},\{|R \downarrow, n\rangle\} \leftrightarrow\{21, \ldots, 25\},\{|R \uparrow, n\rangle\} \leftrightarrow\{26, \ldots, 30\}$, where $n=0, \ldots, 4$ is the index associated with the oscillator states.

similar for small $\varepsilon$, before the first phonon peak. However, for large values of the detuning, we see that while the current in the TQD rapidly drops to zero, in the TQDS it exhibits well defined resonances. These resonances correspond to the opening of new conduction channels induced by vibrational sidebands due to the oscillation of the central dot. To understand the observed features, we explore the energy spectra for one electron in the closed system corresponding to the TQDS and determine the most important state contributions to the satellite peaks induced by SOI. In Fig. 2(a) we show the energy spectrum of the shuttle with homogeneous Zeeman splitting and SOI. Note that each eigenvalue has replicas at energy intervals separated by $\omega=1$, due to the oscillations of the central dot. A slight displacement between replicas is also observed, due to the finite value $N=4$ of oscillator states used in this calculation. In general the spectra with and without SOI almost fully overlap, except in regions corresponding to the satellite peaks in the current, where level anticrossings appear due to the SOI. Indeed, an amplification of the spectrum around the value of $\varepsilon=0.770$ [Fig. 2(b)] shows this behavior. The case without SOI [open (black) circles] is included for comparison, showing a level crossing. We proceed to investigate the components of the eigenvector for one particular eigenvalue, $E=0.498$, computed in Fig. 2(b) and indicated by the black arrow. This value is associated with $\varepsilon=0.77$, which corresponds to the spin-up satellite peak in Fig. 1(c). The histogram in Fig. 2(c) shows the probability density in terms of the components 


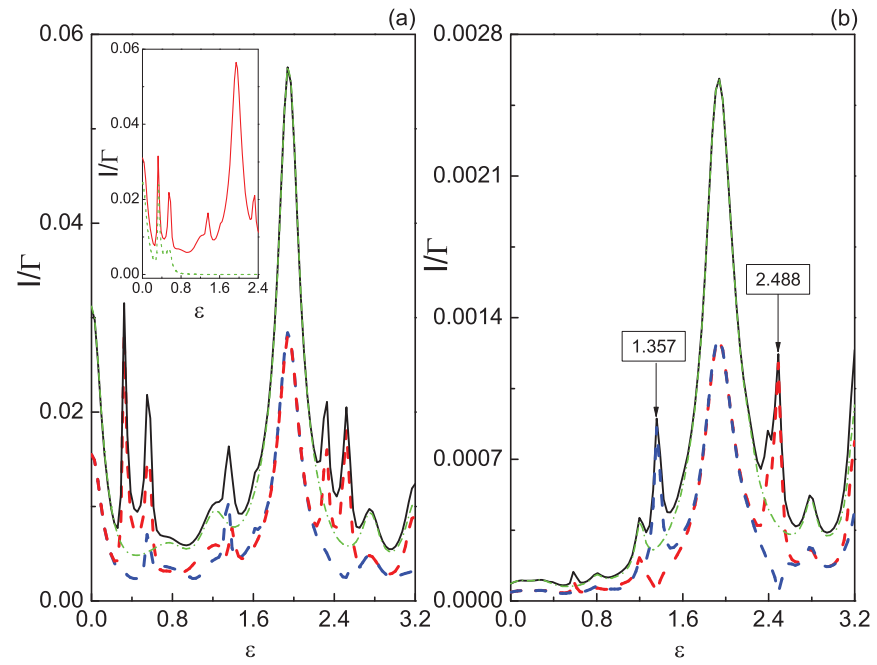

FIG. 3. (Color online) Current through the TQDS [solid (black) line], with SOI and asymmetrical Zeeman splitting $\Delta_{L}=0.2$, $\Delta_{C}=0.4$, and $\Delta_{R}=0.6$, compared to the current with no SOI [dashed-dotted (green) line] in (a) the strong-coupling regime (small $\alpha=0.4$ ), and in (b) the weak-coupling regime (large $\alpha=0.8$ ). In both cases we show the different contributions, spin-up [dashed (blue) line] and spin-down [dashed (red) line], to the SOI current. Note the satellite peaks due to the SOI (see text). Inset in (a): Comparison of the spin current through the TQDS [solid (red) line] in the strong-coupling regime and the static TQD[dashed (green) line] with SOI. Parameter values are the same as in Fig. 1.

of the eigenvector associated with this eigenvalue. We see that the conduction channel is mainly due to the process $|L \downarrow, 0\rangle \rightarrow|C \uparrow, 1\rangle \rightarrow|R \uparrow, 1\rangle$; the referred basis states are $|q \sigma, n\rangle$, where $q=L, C, R$ denotes the electronic states, $\sigma=\uparrow, \downarrow$ represents the spin, and $n=0, \ldots, N$ stands for the oscillator states. Note that a spin flip occurs in the process and that the final state corresponds to a spin-up state. A similar analysis shows that the shoulder at $\varepsilon \simeq 1.178$ corresponds predominantly to a spin-down current (not shown).
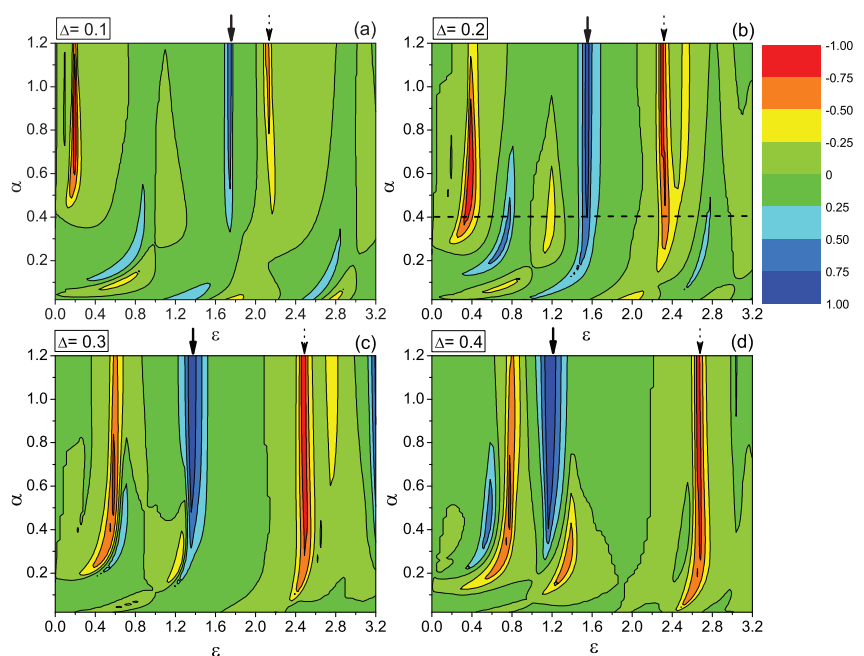

\section{B. Asymmetric case}

Next we discuss the results for the spin current [see Eq. (14)] as a function of the detuning $\varepsilon$ for the TQDS with asymmetric Zeeman splitting. For strong tunneling coupling $(\alpha=0.4)$ [Fig. 3(a)], we see the appearance of new peaks in the current, due to SOI, where the spin-up and spin-down contributions are shown. As in the symmetric case, in the inset in Fig. 3(a) we compare this with results for the static TQD, clearly showing the appearance of new resonances due to the shuttle mechanism. The eigenvectors of the corresponding closed system show a strong mixing of the states involved, and the identification of the conduction channel is less clear. Therefore in order to understand the underlying physical mechanisms we study the weak-coupling regime. This is simply achieved by increasing the value of the parameter $\alpha$. Thus, in Fig. 3(b) for $\alpha=0.8$, we observe clear current peaks at $\varepsilon \approx 1.357$ and $\varepsilon \approx 2.5$, corresponding to spin-up and spin-down contributions, respectively, resulting from the presence of SOI. Note in both cases the pronounced decrease in the current of opposite polarization. An energy spectrum analysis (not shown here) shows that the spin-up current contribution at $\varepsilon \approx 1.357$ comes mainly from the conduction channel $|L \downarrow, 0\rangle \rightarrow|C \uparrow, 1\rangle \rightarrow|R \uparrow, 2\rangle$; similarly, for the peak at $\varepsilon \approx 2.5$, we obtain a spin-down current contribution. Finally, in the case of the large peak at $\varepsilon \approx 2$ (Fig. 3), we can see that there are two conduction channels, with similar characteristics, one for each spin direction.

\section{Spin polarization in a TQDS}

Here, we analyze the effect of SOI on the spin polarization $P$, defined as

$$
P=\left(I^{\uparrow}-I^{\downarrow}\right) /\left(I^{\uparrow}+I^{\downarrow}\right) .
$$

In Figs. 4(a)-4(d), we present contour plots for the TQDS with symmetric Zeeman splitting, as a function of the detuning $\varepsilon$ for a wide range of values of the coupling parameter $\alpha$.
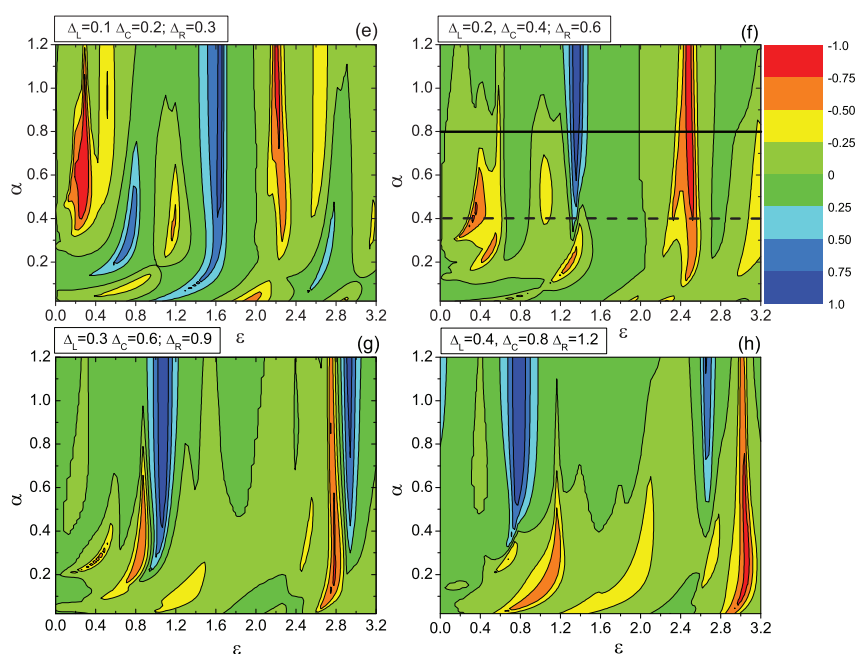

FIG. 4. (Color online) Contour plot of the SOI current polarization $P$ for the TQDS with (a)-(d) symmetric $\left(\Delta=\Delta_{m}, m=L, C, R\right)$ and (e)-(h) asymmetric Zeeman splitting, indicated at the top of each panel as a function of the detuning $\varepsilon$ and coupling parameter $\alpha$. The shades of blue (red) indicated by solid (dotted) arrows at the top of each panel, correspond mainly to spin-up (spin-down) contributions; the shades of green correspond to $P \simeq 0$. Parameter values are the same as in Fig. 1 . 
In this case, we see that as $\alpha$ increases (the tunnel coupling decreases), well-defined regions of high polarization develop due to SOI, with $|P| \simeq 1$. In particular, note the two regions of opposite polarization, shown by solid (spin-up) and dotted (spin-down) arrows, which tend to separate as the Zeeman splitting (shown at the top of each panel) increases. This can be explained by the energy spectrum [Fig. 2(a)], where one can see that as the Zeeman splitting increases, the anticrossing points will be displaced farther to the left and right with respect to each phonon peak. Thus, in this regime of parameters, the TQDS shows a spin filter behavior where peaks of opposite polarization, with $|P| \simeq 1$, appear as a function of detuning. Furthermore, in Fig. 4(b), along the dashed black line at $\alpha=$ 0.4 , high values of the polarization $P$ appear, corresponding to the SOI current peaks presented in Fig. 1(b). Similarly, in Figs. 4(e)-4(h), we explore the behavior of the polarization $P$ for the TQDS with different asymmetric Zeeman splitting configurations. Again, we obtain spin filter behavior, since as $\alpha$ increases, the polarization increases for particular values of detuning, being positive (or negative) for a wide range of values of the parameter $\alpha$. In particular, let us analyze more closely the contour plot in Fig. 4(f), where we see that the spin polarization is stronger at the values of $\varepsilon \simeq 1.36$ and 2.5 , being positive in the vicinity of $\varepsilon \simeq 1.36$ and negative at $\varepsilon \simeq 2.5$, corresponding to the main current peaks induced by SOI, discussed in Figs. 3(a) and 3(b) for different values of $\alpha$. Note also that for $\alpha \lesssim 0.4$, the polarization exhibits predominantly negative values corresponding to a spin-down polarization, which for the present case is a distinctive feature of the strong-coupling regime. We also indicate in Fig. 4(f) the corresponding polarization for the two cases with asymmetric Zeeman splitting analyzed in Figs. 3(a) and 3(b). These cases correspond, respectively, to the values of $\alpha=0.4$ [dashed (black) line] and $\alpha=0.8$ [solid (black) line]. We see that in the weak-tunneling regime $(\alpha=0.8)$, we obtain a spin filter effect as a function of detuning $\varepsilon$, due to both SOI and asymmetrical Zeeman splitting.

\section{CONCLUSIONS}

In summary, the effects of SOI interaction on the spin current through a vibrating TQD system in a linear arrangement has been explored as a function of detuning and tunnel coupling intensity, in the presence of a magnetic field. Using the density matrix master equation approach, we calculate the current and polarization for both static and dynamic conditions, for symmetric and asymmetric Zeeman splitting configurations. We note that the shuttle mechanism (i.e., position-dependent interdot tunneling) gives rise to resonances, where vibrational sidebands allow opening of new conduction channels. Additional resonance peaks are obtained in the presence of SOI. These resonances are shown to correspond to anticrossings in the energy spectrum reflecting coupling between states due to SOI. By calculation of the eigenvectors at these anticrossings, we identify the states involved in the conduction process at each current peak. Finally, for both symmetric and asymmetric TQDS we have carried out a systematic analysis of the current polarization as a function of the tunnel coupling intensity $\alpha$ of the oscillating QD with the outer dots, detuning $\varepsilon$, and Zeeman splitting asymmetry. We have found that the spin current becomes polarized for both weak and strong coupling regimes and identified conditions under which the current is fully polarized presenting a spin filter behavior. To our knowledge, these properties of the spin current, in a system where interdot tunnel coupling is position dependent, including SOI, have not been reported before. We hope that our work provides new insight into the spin transport properties of shuttle semiconductor nanodevices where SOI is important.

\section{ACKNOWLEDGMENTS}

We gratefully acknowledge support from projects UNAM-DGAPA PAPIIT IN112012 (E.C.), P/PIFI 201102MSU0020A-08 (J.V.), MAT 2011-24331 and ITN Grant No. 234970 EU (G.P.).

\footnotetext{
*villavics@uabc.edu.mx

†ernesto@cnyn.unam.mx

†gplatero@icmm.csic.es

${ }^{1}$ L. Gaudreau, S. A. Studenikin, A. S. Sachrajda, P. Zawadzki, A. Kam, J. Lapointe, M. Korkusinski, and P. Hawrylak, Phys. Rev. Lett. 97, 036807 (2006); L. Gaudreau, A. Kam, G. Granger, S. A. Studenikin, P. Zawadzki, and A. S. Sachrajda, Appl. Phys. Lett. 95, 193101 (2009).

${ }^{2}$ D. S. Saraga and D. Loss, Phys. Rev. Lett. 90, 166803 (2003).

${ }^{3}$ M. C. Rogge and R. J. Haug, Phys. Rev. B 77, 193306 (2008).

${ }^{4}$ L. Gaudreau, G. Granger, A. Kam, G. C. Aers, S. A. Studenikin,

P. Sawadzki, M. Pioro-Ladrière, Z. R. Wasilewski, and A. S. Sachrajda, Nat. Phys. 8, 54 (2012).

${ }^{5}$ J. Medford, J. Beil, J. M. Taylor, S. D. Bartlett, A. C. Doherty, E. I. Rashba, D. P. DiVincenzo, H. Lu, A. C. Gossard, and C. M. Marcus, Nat. Nanotechnol. 8, 654 (2013); J. Medford, J. Beil, J. M. Taylor, E. I. Rashba, H. Lu, A. C. Gossard, and C. M. Marcus, Phys. Rev. Lett. 111, 050501 (2013).
}

${ }^{6}$ M. Busl, G. Granger, L. Gaudreau, R. Sánchez, A. Kam, M. PioroLadrière, S. A. Studenikin, P. Zawadzki, Z. R. Wasilewski, A. S. Sachrajda, and G. Platero, Nat. Nanotechnol. 8, 261 (2013).

${ }^{7}$ C.-Y. Hsieh, Y.-P. Shim, and P. Hawrylak, Phys. Rev. B 85, 085309 (2012).

${ }^{8}$ F. Delgado, Y.-P. Shim, M. Korkusinski, and P. Hawrylak, Phys. Rev. B 76, 115332 (2007).

${ }^{9}$ M. Busl, R. Sánchez, and G. Platero, Phys. Rev. B 81, 121306(R) (2010).

${ }^{10}$ M. Busl and G. Platero, Phys. Rev. B 82, 205304 (2010).

${ }^{11}$ J. Villavicencio, I. Maldonado, R. Sánchez, E. Cota, and G. Platero, Appl. Phys. Lett. 92, 192102 (2008); J. Villavicencio, I. Maldonado, E. Cota, and G. Platero, New J. Phys. 13, 023032 (2011).

${ }^{12}$ D. V. Scheible and R. H. Blick, Appl. Phys. Lett. 84, 4632 (2004).

${ }^{13}$ C. Kim, M. Prada, G. Platero, and R. H. Blick, Phys. Rev. Lett. 111, 197202 (2013); C. Kim, M. Prada, and R. H. Blick, ACS Nano 6, 651 (2012). 
${ }^{14}$ A. D. Armour and A. MacKinnon, Phys. Rev. B 66, 035333 (2002).

${ }^{15}$ T. Novotný, A. Donarini, and A.-P. Jauho, Phys. Rev. Lett. 90, 256801 (2003).

${ }^{16}$ A. Donarini, Ph.D. thesis, Technical University of Denmark (2004).

${ }^{17}$ M. Pioro-Ladrière, T. Obata, Y. Tokura, Y.-S. Shin, T. Kubo, K. Yoshida, T. Taniyama, and S. Tarucha, Nat. Phys. 4, 776 (2008).

${ }^{18}$ S. M. Huang, Y. Tokura, H. Akimoto, K. Kono, J. J. Lin, S. Tarucha, and K. Ono, Phys. Rev. Lett. 104, 136801 (2010).

${ }^{19}$ J. López-Bonilla and G. Ovando, Bull. Irish Math. Soc. 44, 61 (2000); J. Morales, J. López-Bonilla, and A. Palma, J. Math. Phys. 28, 1032 (1987).

${ }^{20}$ T. H. Stoof and Yu. V. Nazarov, Phys. Rev. B 53, 1050 (1996).
${ }^{21}$ F. Mireles and G. Kirczenow, Phys. Rev. B 64, 024426 (2001).

${ }^{22}$ J. Danon and Y. V. Nazarov, Phys. Rev. B 80, 041301(R) (2009).

${ }^{23}$ D. V. Khomitsky, L. V. Gulyaev, and E. Ya. Sherman, Phys. Rev. B 85, 125312 (2012).

${ }^{24}$ F. Baruffa, P. Stano, and J. Fabian, Phys. Rev. B 82, 045311 (2010).

${ }^{25}$ A. Pfund, I. Shorubalko, K. Ensslin, and R. Leturcq, Phys. Rev. Lett. 99, 036801 (2007).

${ }^{26} \mathrm{~K}$. Blum, Density Matrix Theory and Applications (Plenum Press, New York, 1996).

${ }^{27}$ C. Cohen-Tannoudji, J. Dupont-Roc, and G. Grynberg, AtomPhoton Interactions: Basic Processes and Applications (John Wiley \& Sons, New York, 1993).

${ }^{28}$ M. O. Scully and M. S. Zubairy, Quantum Optics (Cambridge University Press, Cambridge, 1997). 